ANNALES

POLONICI MATHEMATICI

XXVI (1972)

\title{
An application of a functional equation to information theory
}

by P. Kannappan and P. N. Rathie (Waterloo)

1. Introduction. Let $P=\left(p_{1}, \ldots, p_{n}\right), \quad p_{i} \geqslant 0, \sum_{i=1}^{n} p_{i}=1, \quad Q=$ $=\left(q_{1}, \ldots, q_{n}\right), q_{i} \geqslant 0, \sum_{i=1}^{n} q_{i}=1$ and $R=\left(r_{1}, \ldots, r_{n}\right), \quad r_{i} \geqslant 0, \sum_{i=1}^{n} r_{i}=1$ be three finite discrete probability distributions. Then we define a generalized directed-divergence function of type $\beta(\beta \neq 1)$ and a generalized directed-divergence of type $\beta(\beta \neq 1)$ as follows:

Definition 1. A real valued function $f$ defined on $I \times I \times I$, where $I=[0,1]$, is called a generalized directed-divergence function of type $\beta$ $(\neq 1)$ if $f$ is a solution of the functional equation,

$$
\begin{aligned}
& f(x, y, z)+(1-x)(1-y)^{\beta-1}(1-z)^{1-\beta} f\left(\frac{u}{1-x}, \frac{v}{1-y}, \frac{w}{1-z}\right) \\
& =f(u, v, w)+(1-u)(1-v)^{\beta-1}(1-w)^{1-\beta} f\left(\frac{x}{1-u}, \frac{y}{1-v}, \frac{z}{1-w}\right)
\end{aligned}
$$

for $x, y, z, u, v, w \in[0,1), x+u, y+v, z+w \in I$, satisfying further

$$
f(0,0,0)=f(1,1,1)
$$

and

$$
f\left(1,1, \frac{1}{2}\right)=f\left(0,0, \frac{1}{2}\right)=1 .
$$

DEFINITION 2. If $f$ is a generalized directed-divergence function of type $\beta(\neq 1)$ as defined above, then the generalized directed-divergence of type $\beta(\neq 1)$ is defined by the relation

$$
I_{n}^{\beta}\left(\begin{array}{ccc}
p_{1} & \ldots & p_{n} \\
q_{1} & \ldots & q_{n} \\
r_{1} & \ldots & r_{n}
\end{array}\right) \stackrel{\mathrm{d} P}{=} \sum_{i=2}^{n} P_{i} Q_{i}^{\beta-1} R_{i}^{1-\beta} f\left(\frac{p_{i}}{P_{i}}, \frac{q_{i}}{Q_{i}}, \frac{r_{i}}{R_{i}}\right),
$$

where $P_{i}=p_{1}+\ldots+p_{i}, \quad Q_{i}=q_{1}+\ldots+q_{i}, \quad R_{i}=r_{1}+\ldots+r_{i}$ for $i=1,2, \ldots, n$ with $P_{n}=Q_{n}=R_{n}=1$. 
The object of this paper is to solve the functional equation (1) and to find $I_{n}^{\beta}$ and to discuss some connection of these quantities with the existing measures of information. This has been done in subsequent sections to follow. The case $\beta=1$ will be discussed by us elsewhere.

2. This section deals with the solution of the functional equation (1) under the boundary conditions (2) and (3) and then to utilize this solution in finding the expression of $I_{n}^{\beta}$.

THEOREM 1. If $f$ is a solution of the functional equation (1) satisfying the additional conditions (2) and (3), then $f$ has the form

$$
f(x, y, z)=\left[x y^{\beta-1} z^{1-\beta}+(1-x)(1-y)^{\beta-1}(1-z)^{1-\beta}-1\right]\left(2^{\beta-1}-1\right)^{-1}
$$

for all $x, y, z \in I$ and conversely.

Remark. In (5) we use the convention $0^{a}=0(a \neq 0)$ but nowhere in the proof of the theorem $0^{a}=0$ is used. Only to put the solution of (1) in the form (5), this notation is used.

Proof. Taking $x=0, y=0, z=0$ in (1) gives $f(0,0,0)=0$ and hence from (2), we get

$$
f(1,1,1)=f(0,0,0)=0 .
$$

Now replacing $u, v, w$ in (1) by $1-x, 1-y$ and $1-z$ respectively and applying (6), we have

$$
f(x, y, z)=f(1-x, 1-y, 1-z) \quad \text { for } x, y, z \epsilon(0,1) .
$$

With $p=\frac{u}{1-x}, q=\frac{v}{1-y}, r=\frac{w}{1-z}, \xi=1-x, \eta=1-y$ and $\zeta=1-z$ in (1), (1) takes the form

(8) $f(1-\xi, 1-\eta, 1-\zeta)+\xi \eta^{\beta-1} \zeta^{1-\beta} f(p, q, r)$

$$
=f(p \xi, q \eta, r \zeta)+(1-p \xi)(1-q \eta)^{\beta-1}(1-r \zeta)^{1-\beta} \times f\left(\frac{1-\xi}{1-p \xi}, \frac{1-\eta}{1-q \eta}, \frac{1-\zeta}{1-r \zeta}\right)
$$

for all $p, q, r \in I$ and $\xi, \eta, \zeta \in(0,1]$ such that $p \xi \neq 1, q \eta \neq 1$ and $r \zeta \neq 1$.

Putting $\xi=1, \eta=1, \zeta=\frac{1}{2}, r=0$ in (8) and using (3), we get

$$
f(p, q, 0)=\left[(1-p)(1-q)^{\beta-1}-1\right]\left(2^{\beta-1}-1\right)^{-1} \quad \text { for } p, q \in[0,1) \text {. }
$$

Taking $\xi=1, \eta=\frac{1}{2}, \zeta=1$ and $q=0$ in (8) and using (9) for $p=0$ and $q=\frac{1}{2}$, we have

$$
f(p, 0, r)=\left[(1-p)(1-r)^{1-\beta}-1\right]\left(2^{\beta-1}-1\right)^{-1} \quad \text { for } p, r \in[0,1) .
$$

Again, taking $\xi=\frac{1}{2}, \eta=1, \zeta=1$ and $p=0$ in (8) and utilizing (9) we get

(11) $f(0, q, r)=\left[(1-q)^{\beta-1}(1-r)^{1-\beta}-1\right]\left(2^{\beta-1}-1\right)^{-1}$ for $q, r \in[0,1)$. 
With $p=1, q=1$ and $r=0,(8)$ becomes

$$
\begin{aligned}
& f(1-\xi, 1-\eta, 1-\zeta)+\xi \eta^{\beta-1} \zeta^{1-\beta} f(1,1,0)=f(\xi, \eta, 0)+ \\
& \quad+(1-\xi)(1-\eta)^{\beta-1} f(1,1,1-\zeta) \quad \text { for } \xi, \eta \epsilon(0,1), \zeta \epsilon(0,1] .
\end{aligned}
$$

Taking $\zeta=1$ in (12), in view of (9), we obtain that

$$
f(1,1,0)=\left(1-2^{\beta-1}\right)^{-1} \text {. }
$$

Putting $p=0, q=0$ and $r=1$ in (8), we get

$$
\begin{aligned}
& f(1-\xi, 1-\eta, 1-\zeta)+\xi \eta^{\beta-1} \zeta^{1-\beta} f(0,0,1)=f(0,0, \zeta)+ \\
& +(1-\zeta)^{1-\beta} f(1-\xi, 1-\eta, 1) \text { for } \xi, \eta \in(0,1], \zeta \in(0,1) .
\end{aligned}
$$

Setting $\xi=1, \eta=1$ in (14) and making use of (10), we have

$$
f(0,0,1)=\left(1-2^{\beta-1}\right)^{-1} \text {. }
$$

For $\zeta=\frac{1}{2}$ in (12) and using (3), (9) and (13) respectively, (12) gives

$$
f\left(1-\xi, 1-\eta, \frac{1}{2}\right)=\left[2^{\beta-1} \xi \eta^{\beta-1}+2^{\beta-1}(1-\xi)(1-\eta)^{\beta-1}-1\right]\left(2^{\beta-1}-1\right)^{-1}
$$$$
\text { for } \xi, \eta \in(0,1) \text {. }
$$

Now $\zeta=\frac{1}{2}$ in (14), with the help of (3), (15) and (16), gives

$$
f(1-\xi, 1-\eta, 1)=\left[(1-\xi)(1-\eta)^{\beta-1}-1\right]\left(2^{\beta-1}-1\right)^{-1}
$$

$$
\text { for } \xi, \eta \in(0,1) \text {. }
$$

Hence, (10), (14), (15) and (17) give us

$$
\begin{aligned}
& f(1-\xi, 1-\eta, 1-\zeta)=\left[\xi \eta^{\beta-1} \zeta^{1-\beta}+\right. \\
& \left.+(1-\xi)(1-\eta)^{\beta-1}(1-\zeta)^{1-\beta}-1\right]\left(2^{\beta-1}-1\right)^{-1} \quad \text { for } \xi, \eta, \zeta \epsilon(0,1) .
\end{aligned}
$$

With $p=1, q=0, r=0,(8)$ can be written as

$$
\begin{aligned}
& f(1-\xi, 1-\eta, 1-\zeta)+\xi \eta^{\beta-1} \zeta^{1-\beta} f(1,0,0)=f(\xi, 0,0)+ \\
& \quad+(1-\xi) f(1,1-\eta, 1-\zeta) \text { for } \xi \epsilon(0,1), \eta, \zeta \epsilon(0,1] .
\end{aligned}
$$

Taking $\eta=1, \zeta=1$ in (19) and using (9), we get

$$
f(1,0,0)=\left(1-2^{\beta-1}\right)^{-1} \text {. }
$$

With $p=1, q=0, r=1$, (8) becomes

$$
\begin{aligned}
& f(1-\xi, 1-\eta, 1-\zeta)+\xi \eta^{\beta-1} \zeta^{1-\beta} f(1,0,1)=f(\xi, 0, \zeta)+ \\
& +(1-\xi)(1-\zeta)^{1-\beta} f(1,1-\eta, 1) \quad \text { for } \xi, \zeta \epsilon(0,1) \text { and } \eta \epsilon(0,1] .
\end{aligned}
$$

Taking $\eta=1$ in (21) and using (10) we have

$$
f(1,0,1)=\left(1-2^{\beta-1}\right)^{-1} .
$$

7 - Annales Polonici Mathematici XXVI 
With $p=0, q=1, r=1,(8)$ becomes

$$
\begin{aligned}
& f(1-\xi, 1-\eta, 1-\zeta)+\xi \eta^{\beta-1} \zeta^{1-\beta} f(0,1,1)=f(0, \eta, \zeta)+ \\
& +(1-\eta)^{\beta-1}(1-\zeta)^{1-\beta} f(1-\xi, 1,1) \text { for } \xi \epsilon(0,1], \eta, \zeta \epsilon(0,1) .
\end{aligned}
$$

For $\xi=1$ in (23) and utilizing (11), we get

$$
f(0,1,1)=\left(1-2^{\beta-1}\right)^{-1} .
$$

Taking $p=0, q=1$ and $r=1$ in (8) we have

$$
\begin{aligned}
& f(1-\xi, 1-\eta, 1-\zeta)+\xi \eta^{\beta-1} \zeta^{1-\beta} f(0,1,0)=f(0, \eta, 0)+ \\
& \quad+(1-\eta)^{\beta-1} f(1-\xi, 1,1-\zeta) \text { for } \xi, \zeta \epsilon(0,1], \eta \epsilon(0,1) .
\end{aligned}
$$

Putting $\xi=1, \zeta=1$ in (25) and using (9) with $p=0$, we get

$$
f(0,1,0)=\left(1-2^{\beta-1}\right)^{-1} \text {. }
$$

Now (23) by the help of (18), (11) and (24) gives

$$
f(1-\xi, 1,1)=\xi\left(1-2^{\beta-1}\right)^{-1} \quad \text { for } \xi \epsilon(0,1) .
$$

On using (26), (9) and (18), (25) gives

$$
\begin{aligned}
& f(1-\xi, 1,1-\zeta)=\left[(1-\xi)(1-\zeta)^{1-\beta}-1\right]\left(2^{\beta-1}-1\right)^{-1} \\
& \quad \text { for } \xi, \zeta \epsilon(0,1) .
\end{aligned}
$$

Equation (19) with the help of (9), (18) and (20) gives

$$
\begin{aligned}
& f(1,1-\eta, 1-\zeta)=\left[(1-\eta)^{\beta-1}(1-\zeta)^{1-\beta}-1\right]\left(2^{\beta-1}-1\right)^{-1} \\
& \text { for } \eta, \zeta \epsilon(0,1) .
\end{aligned}
$$

Now (12), (13), (9) and (18) imply

$$
f(1,1,1-\zeta)=\left[(1-\zeta)^{1-\beta}-1\right]\left(2^{\beta-1}-1\right)^{-1} \quad \text { for } \zeta \epsilon(0,1) .
$$

In (21), on using (18), (10) and (22), we obtain

$$
f(1,1-\eta, 1)=\left[(1-\eta)^{\beta-1}-1\right]\left(2^{\beta-1}-1\right)^{-1} \quad \text { for } \eta \epsilon(0,1) .
$$

With $\zeta=1$ in (19) and utilizing (20) and (9), (19) gives

$$
f(1,1-\eta, 0)=\left(1-2^{\beta-1}\right)^{-1} \quad \text { for } \eta \epsilon(0,1] \text {. }
$$

Also (19) with $\eta=1$ on utilizing (20) and (10) gives

$$
f(1,0,1-\zeta)=\left(1-2^{\beta-1}\right)^{-1} \quad \text { for } \zeta \epsilon(0,1] .
$$

Taking $\eta=1$ in (14) with (15) and (10) gives

$$
f(1-\xi, 0,1)=\left(1-2^{\beta-1}\right)^{-1} \quad \text { for } \xi \in(0,1],
$$


while $\xi=1$ in (14) with (15) and (11) gives

$$
f(0,1-\eta, 1)=\left(1-2^{\beta-1}\right)^{-1} \quad \text { for } \eta \in(0,1] \text {. }
$$

Also (25) for $\xi=1$ on using (26) and (11) gives

$$
f(0,1,1-\zeta)=\left(1-2^{\beta-1}\right)^{-1} \quad \text { for } \zeta \epsilon(0,1],
$$

while (25) for $\zeta=1$ on utilizing (26) and (9) gives

$$
f(1-\xi, 1,0)=\left(1-2^{\beta-1}\right)^{-1} \quad \text { for } \xi \epsilon(0,1]
$$

Combining (18), (6), (7), (9), (10), (11), (13), (15), (17), (20), (22). (24) and (26) to (37), we conclude that $f$ is given by (5) for all $x, y, z \in I$,

It is easy to verify the converse part by straightforward substitution.

Theorem 2. The generalized directed-divergence of type $\beta(\neq 1)$ is given by

$$
I_{n}^{\beta}\left(\begin{array}{lll}
p_{1} & \ldots & p_{n} \\
q_{1} & \ldots & q_{n} \\
r_{1} & \ldots & r_{n}
\end{array}\right)=\left(\sum_{i=1}^{n} p_{i} q_{i}^{\beta-1} r_{i}^{1-\beta}-1\right)\left(2^{\beta-1}-1\right)^{-1}
$$

Proof. Substituting the expression for $f$ from (5) in (4) we have

$$
\begin{aligned}
I_{n}^{\beta}\left(\begin{array}{lll}
p_{1} & \ldots & p_{n} \\
q_{1} & \ldots & q_{n} \\
r_{1} & \ldots & r_{n}
\end{array}\right) & =\left(2^{\beta-1}-1\right)^{-1} \sum_{i=2}^{n}\left[p_{i} q_{i}^{\beta-1} r_{i}^{1-\beta}+P_{i-1} Q_{i-1}^{\beta-1} R_{i-1}^{1-\beta}-P_{i} Q_{i}^{\beta-1} R_{i}^{1-\beta}\right] \\
& =\left(2^{\beta-1}-1\right)^{-1}\left[\sum_{i=2}^{n} p_{i} q_{i}^{\beta-1} r_{i}^{1-\beta}+P_{1} Q_{1}^{\beta-1} R_{1}^{1-\beta}-P_{n} Q_{n}^{\beta-1} R_{n}^{1-\vartheta}\right] \\
& =\left(2^{\beta-1}-1\right)^{-1}\left[\sum_{i=1}^{n} p_{i} q_{i}^{\beta-1} r_{i}^{1-\beta}-1\right],
\end{aligned}
$$

which is (38).

3. Applications to information theory. This section deals with the connection of $I_{n}^{\beta}$ defined in this paper with the existing measures of information.

The expression (38) for $\beta \rightarrow 1$ gives

$$
I_{n}^{1}\left(\begin{array}{ccc}
p_{1} & \ldots & p_{n} \\
q_{1} & \ldots & q_{n} \\
r_{1} & \ldots & r_{n}
\end{array}\right)=\sum_{i=1}^{n} p_{i} \log \left(q_{i} / r_{i}\right)
$$


which is called the generalized directed-divergence of type unity. Here the convention $0 \log 0=0$ is followed. Also whenever $q_{i}$ or $r_{i}$ is zero then the corresponding $p_{i}$ is also zero and $\log \left(q_{i} / r_{i}\right)$ is to be taken as $\left(\log q_{i}-\log r_{i}\right)$ Also (39) for $P \equiv Q$ gives the well-known directed-divergence [2],

$$
I_{n}^{1}\left(\begin{array}{ccc}
p_{1} & \ldots & p_{n} \\
p_{1} & \ldots & p_{n} \\
r_{1} & \ldots & r_{n}
\end{array}\right)=\sum_{i=1}^{n} p_{i} \log \left(p_{i} / r_{i}\right)
$$

Generalized case of finite discrete probability distributions $P=\left(p_{1}\right.$, $\left.\ldots, p_{n}\right), \quad p_{i}>0, \quad \sum_{i=1}^{n} p_{i} \leqslant 1, \quad Q=\left(q_{1}, \ldots, q_{n}\right), \quad q_{i}>0, \quad \sum_{i=1}^{n} q_{i} \leqslant 1$ and $R$ $=\left(r_{1}, \ldots, r_{n}\right), r_{i}>0, \sum_{i=1}^{n} r_{i} \leqslant 1$, two measures of generalized directeddivergences are defined in [1] as follows:

(42) $I_{a}(P|| Q \mid R)=(a-1)^{-1} \log _{2}\left[\sum_{i=1}^{n} p_{i} q_{i}^{a-1} r_{i}^{1-a} / \sum_{i=1}^{n} p_{i}\right] \quad$ for $a \neq 1$.

The motivation for considering (41) and (42) and some axiomatic characterizations are discussed in [1]. relation

Clearly $I_{n}^{\beta}$ given by (38), and $I_{a}$ given by (42) are connected by the

$$
I_{n}^{\beta}\left(\begin{array}{lll}
p_{1} & \ldots & p_{n} \\
q_{1} & \ldots & q_{n} \\
r_{1} & \ldots & r_{n}
\end{array}\right)=\left(2^{\beta-1}-1\right)^{-1}\left[2^{(\beta-1) I_{\beta}(P|| Q \mid R)}-1\right]
$$

for $\sum_{i=1}^{n} p_{i}=\sum_{i=1}^{n} q_{i}=\sum_{i=1}^{n} r_{i}=1$. Therefore the results of this paper also give alternative characterization of (42) for complete probability distributions.

It is interesting to note that for $P \equiv Q,(38)$ reduces to

$$
I_{n}^{\beta}\left(\begin{array}{lll}
p_{1} & \ldots & p_{n} \\
p_{1} & \ldots & p_{n} \\
r_{1} & \ldots & r_{n}
\end{array}\right)=\left(2^{\beta-1}-1\right)^{-1}\left(\sum_{i=1}^{n} p_{i}^{\beta} r_{i}^{1-\beta}-1\right), \quad \beta \neq 1,
$$

which is called a directed-divergence of type $\beta$ and is recently discussed by us in [3]. This quantity (44) has a connection with Rényi's information gain of order $\beta$ [4]. In our paper [3], a new definition of the directeddivergence function of type $\beta$ is given and characterized by means of a functional equation. 


\section{References}

[1] J. Aczél and P. Nath, Axiomatic characterizations of some measures of divergence in information, 4th Hawaii International Conference on System Sciences, Univ. of Hawaii, Honolulu, January 1971.

[2] S. Kullback, Information theory and statistics, New York 1959.

[3] P. N. Rathie and P. L. Kannappan, $A$ directed-divergence function of type $\beta$ (to appear in Information and Control).

[4] A. Rónyi, On measures of entropy and information, Proc. Fourth Berkeley Symposium Math. Statist. and Probability, 1 (1961), p. 547-561.

UNIVERSITY OF WATERLOO

WATERLOO

Reģu par la Rédaction le 25. 3. 1971 